\title{
Foreword
}

\section{Craniovertebral Junction Lesions (Part 1)}

James K. Liu, MD ${ }^{1,2}$

\footnotetext{
${ }^{1}$ Department of Neurological Surgery, Rutgers University, New Jersey Medical School, Newark, New Jersey, United States

2 Department of Otolaryngology-Head and Neck Surgery, Rutgers, Neurological Institute of New Jersey, Rutgers University-New Jersey, Medical School, Newark, New Jersey, United States
}

J Neurol Surg B 2019;80(suppl S4):S339-S340.

The craniovertebral junction is a unique location in the skull base. A variety of neoplastic and vascular pathologies can arise here including foramen magnum meningiomas, clivus chordomas, jugular foramen schwannomas, glomus jugulare tumors, neurenteric cysts, and posterior inferior cerebellar artery aneurysms. In addition, nonneoplastic conditions, such as basilar invagination, Klippel-Feil syndrome, and rheumatoid pannus can result in neurologic compression of the cervicomedullary junction. In this new issue Craniovertebral Junction Lesions (Part 1) of Skull Base: Operative Videos, we present a collection of videos demonstrating surgical approaches to these pathologies. The approaches can be categorized into anterior (extended endoscopic endonasal), anterolateral, posterolateral (far lateral transcondylar), and posterior (midline suboccipital). The location, extent, and type of pathology, as well as the surgeon's preference, generally determine the choice of approach. It is also important to preserve lower cranial nerve function as postoperative complications of dysphagia and dysphonia that can impact quality of life. Craniocervical instability, whether it is caused by the pathology or iatrogenically by bone removal, needs to be carefully assessed for the need of stabilization. A variety of surgical approaches, operative techniques, and strategies to the craniovertebral junction are demonstrated in this issue of Skull Base: Operative Videos. The link to view the complete collection of operative videos can be found at: www. thieme.com/skullbasevideos.
Address for correspondence James K. Liu, MD, Department of Neurological Surgery, Rutgers University, New Jersey Medical School, 90 Bergen Street, Suite 8100, Newark, NJ 07103, United States (e-mail: james.liu. md@rutgers.edu).
DOI https://doi.org/

10.1055/s-0039-3400246. ISSN 2193-6331.
(C) 2019 Georg Thieme Verlag KG Stuttgart · New York

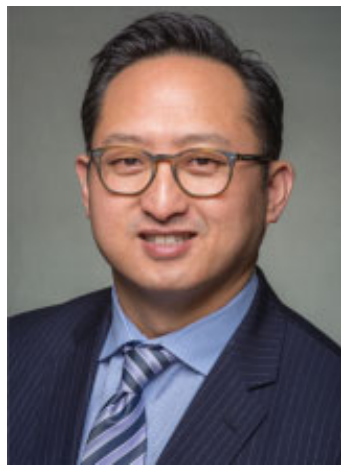

James K. Liu, MD 